\title{
La recherche en monde pilarisé
}

\author{
Socio-histoire de la sexualité dans \\ des institutions catholiques belges
}

\author{
Lynn Bruyère ${ }^{1}, \overline{\text { Anne-Sophie Crosetti }}{ }^{2}$
}

\begin{abstract}
[Résumé] L'objectif de cet article est d'interroger les effets de l'assimilation institutionnelle de chercheur.e.s à une position philosophique laïque dans une recherche qui traite de la gestion de la sexualité par des institutions catholiques - à savoir les plannings familiaux et les institutions de protection de la jeunesse. La structure particulière de la Belgique, reposant sur une société divisée entraînant la création de « piliers » évoluant dans un même milieu, nécessite un retour réflexif sur l'accès au terrain et les conséquences sur la recherche lorsqu'il s'agit de lier étude de la religion et de la sexualité. Comment contourner/dépasser cet étiquetage institutionnel dans la recherche et que nous révèle-t-il sur la logique de (dé)pilarisation en Belgique? L'analyse suivante repose sur les situations d'entretiens (exploratoires et qualitatifs) que nous avons vécues au cours de la première année de recherche doctorale.

Mots-clés : (dé)pilarisation, institutions catholiques, sexualité, étiquetage, stratégie d'accès au terrain.
\end{abstract}

[Abstract] Focused on both Catholic planned parenthood and institutions for children "in danger", this article addresses the effects of the study of sexuality management by Catholic institutions. It highlights the institutional assimilation of researchers to a laic position. The particular structure of Belgium, which is based on a divided society that leads to the creation of "pillars", requires reflection on the way we access our fieldwork since we don't belong to the same "pillar". When working on sexuality and religion, one also needs to question the consequences of such a labelling. How can we bypass institutional labelling and what does it reveal on Belgian (de)pillarization? The analysis will be based on interviews that were lead during the first year of our doctoral research.

Keywords: (de)pillarization, catholic institutions, sexuality, labelling, negotiation and access to fieldwork.

\section{Introduction}

La «pilarisation» constitue une des caractéristiques fondamentales de la société belge, se traduisant par la construction de milieux sociaux séparés (les mondes catholique, laïque et socialiste) encadrant les individus du berceau à la tombe. Ainsi, il était

\footnotetext{
${ }^{1}$ Université libre de Bruxelles.

${ }^{2}$ FNRS/Université libre de Bruxelles.
} 
tout à fait usuel pour un enfant de naitre au sein d'une famille dite catholique, de mener sa scolarité au sein d'écoles catholiques et d'être engagé dans des associations catholiques sans jamais être socialisé dans les autres piliers, dont les membres avaient le même cheminement (Martiniello, Réa, 2003: 261). Depuis les années 1990, se pose la question de la dépilarisation (notamment Dobbelaere, Voyé, 1990), à savoir une reconfiguration des piliers. L'individualisation de la société aurait ainsi mené à cette restructuration du fait d'une transformation des pratiques religieuses, sexuelles et de genre (CRISP, 1974 ; Dobbelaere, Voyé, 2012 ; De Maeyer, Put, Roegiers, Thion, Vanden Bosch, 2006; Houdailles, 1980) dans la deuxième moitié du XXe siècle. Afin d'étudier comment les institutions catholiques et leurs acteurs ont intégré, entre 1960 et 2000, les questions portant sur les recompositions familiales, la mixité scolaire, la contraception et l'avortement, le sida et l'homosexualité, un projet de recherche interdisciplinaire a été élaboré à l'Université libre de Bruxelles (ULB), dont nous faisons partie en qualité de doctorantes. Nos deux thèses s'intéressent à la gestion de la sexualité (De Ganck, D’Hooghe, 2013) au sein d'organisations issues du pilier catholique en Belgique.

La thèse d'Anne-Sophie Crosetti porte sur les plannings familiaux catholiques. Les catholiques, à l'instar des libéraux et des socialistes, ont participé à l'institutionnalisation des centres de consultations conjugales et des plannings familiaux, dont les premiers développements se déploient du milieu des années $1950 \mathrm{au}$ début des années 1970. La FBCCC (Fédération belge des centres de consultations conjugales), le Cefa (Centre d'éducation à la famille et à l'amour), les centres pluralistes ainsi que certaines personnalités, telles que le chanoine Pierre de Locht, ont rendu possible la naissance de la planification familiale catholique. La thèse de Lynn Bruyère étudie l'évolution des pratiques d'encadrement moral des mineurs dits « en danger» au sein d'institutions catholiques de protection de la jeunesse. Ces recherches permettront d'en savoir davantage sur la construction et la mise en pratique des normes sexuelles au sein de ces lieux de vie d'origine catholique. S'inscrivant dans les champs d'étude sur la religion et le genre (Noonan, 1965; Sèvegrand, 1995; McClory, 1998; Flandrin, 2006; Langlois, 2010 ; Mulder, 2013 ; Rochefort, Sanna, 2013 ; Paternotte, Piette, van der Dussen, 2016 ; Bracke, Paternotte, 2016), ces deux recherches se proposent de pallier la méconnaissance du rôle des catholiques dans la planification familiale et dans la normativité sexuelle au sein d'institutions catholiques de protection de la jeunesse.

Même si le terrain que nous étudions s'avère différent, nos deux recherches articulent sexualité et religion. En tant que doctorantes travaillant au sein d'une institution laïque ${ }^{3}$ - l'Université libre de Bruxelles - étudier des institutions catholiques dans un

${ }^{3}$ Conformément à l'usage attesté en Belgique, nous utilisons l'orthographe «laïque » pour parler des piliers non confessionnels (Defosse, 2005 : 183-184); « laïc » renvoyant aux personnes non ecclésiastiques. 
contexte historique de pilarisation nécessite une analyse réflexive encore plus approfondie que de coutume sur la méthodologie et l'entrée sur le terrain. Faire une sociohistoire (Deloye, 2007; Noiriel, 2008) des institutions aide à comprendre un monde en changement. Utiliser les sources orales permet d'enrichir les données issues de sources historiques en donnant plus de poids à l'expérience vécue par les acteurs: «l'entretien [...] est le plus souvent utilisé comme un pis-aller, une manière d'obtenir des informations et points de vue sur un objet que l'on ne peut pas matériellement recueillir in situ par observation directe» (Beaud, 1996: 236). Cet article repose sur un ensemble de douze entretiens, dont la durée varie entre une heure et trois heures, menés pendant la première année de thèse : quatre entretiens informatifs ont été réalisés avec des personnes travaillant dans des institutions ou organisations historiquement rattachées au pilier catholique dont trois d'entre elles ont également un lien avec le milieu féministe. Les huit autres entretiens, qualitatifs, ont été menés auprès de professionnels actifs durant la période étudiée, soit entre 1960 et 2000. Ces derniers travaillent dans le monde catholique, se définissent comme «chrétiens » et défendent des valeurs chrétiennes tout en ayant un regard critique sur le catholicisme. Les enquêtés ont été identifiés et approchés de diverses manières selon les opportunités et la méthode "boule de neige »: après chaque entrevue, la personne interrogée nous dirige vers de potentiels enquêtés qui nous renvoient à leur tour vers d'autres personnalités. En effectuant un retour critique sur ce qui se déroule en amont de ces entretiens et de la consultation des archives ainsi que sur la constitution de nos objets de recherche, cette contribution a pour ambition de mettre en évidence les effets de projections réciproques entre enquêteurs et enquêtés, soit ici entre individus perçus comme appartenant à des mondes idéologiques séparés. Comment dépasser la logique de l'assimilation, qu'elle soit institutionnelle ou philosophique, dans un travail qui traite de la prise en charge de la sexualité par des institutions catholiques ?

Appartenant via notre institution de rattachement au pilier lä̈que, nous sommes en dehors du monde catholique que nous étudions mais en interaction avec celui-ci puisque nous sommes assignées à une identité qui se définit en partie par rapport à ce monde-là. Pour tenter de comprendre les enjeux autour d'un terrain qui nous positionne donc à la fois comme juge et partie, nous reviendrons d'abord sur la structure institutionnelle particulière de la Belgique. Nous présenterons ensuite les conséquences empiriques de l'objet étudié sur le terrain. Enfin, nous tenterons de montrer qu'il est possible de dépasser cette logique de pilarisation tout en se servant de ses effets sur le terrain pour approfondir notre analyse.

\section{La particularité institutionnelle de la Belgique}

Faire une socio-histoire d'une institution, quelle qu'elle soit, nécessite de revenir sur le contexte dans lequel elle est née. L'histoire particulière de la Belgique a façonné son paysage institutionnel. En effet, l'indépendance de la Belgique en 1830 laissait déjà 
présager des divisions: majoritairement néerlandophone, la langue française s'impose malgré tout comme la langue politique du pays. La société belge, pour reprendre les termes d'Arend Lijphart (1977), est une société "plurielle» et «segmentée ». Elle a été historiquement construite dès le début du XIXe siècle sur les clivages sur les clivages confessionnels (et philosophiques) et socio-économiques. Ces divisions n'ont pourtant pas empêché la Belgique d'asseoir une démocratie consociative (Lijphart, 1977), à savoir une démocratie qui reconnait ces divisions et se construit sur ces dernières. Cette structure particulière est aussi appelée pilarisation - concept venant des Pays-Bas et emprunté au néerlandais «verzuiling». Ce système d'organisation politique et social, renvoie à la gestion de cette diversité. Une société basée sur la pilarisation entraîne donc la reconnaissance de groupes (aussi appelés "piliers ») évoluant dans leur propre milieu social et institutionnel partageant une même tendance idéologique. La Belgique compte dans les années 1960 trois piliers: les libéraux, les catholiques et les socialistes. Ces piliers sont à la fois porteurs des institutions qui les représentent et symboles des frontières philosophiques du pays. Malgré quelques alliances politiques, les piliers restent néanmoins organisés selon des valeurs jugées antagonistes :

[...] quand naît dans une localité une organisation liée à un pilier, les deux autres piliers peuvent difficilement rester indifférents, au risque de voir échapper à leur influence sociologique et idéologique une portion importante de la population de cette localité. La notion de rivalité est donc intrinsèquement liée à la pilarisation. (Faniel, Vandaele, 2011:7)

L'Université libre de Bruxelles est un symbole de la structure et de la dynamique de pilarisation. Fondée en 1834 par Pierre-Théodore Verhaegen, franc-maçon et libéral, l'Université a été érigée en réaction à la réinstallation de l'Université catholique de Louvain (Daled, 2009). Les libéraux estiment alors néfaste que les catholiques aient le monopole de l'enseignement dans une période marquée par l'encyclique Mirari Vos $(1832)^{4}$ qui rejette l'idée même de liberté de conscience. Ils souhaitent, à l'inverse, créer un environnement scientifique autonome: «la liberté de l'enseignement était donc "l'indépendance tant à l'égard du pouvoir religieux qu'à l'égard du pouvoir politique" » écrit Pierre Daled (Daled, 2009: 112) en citant Verhaegen. L’Université ne se revendique pas, à sa création, antireligieuse, mais conteste le pouvoir arbitraire du clergé et son emprise sur l'enseignement. Si ses fondateurs, libéraux et francsmaçons, étaient anticléricaux, ils n'en étaient pas moins spiritualistes, croyants et même, pour certains, catholiques pratiquants. Ce n'est que par la suite, à travers quelques crises, que se dégage progressivement la notion de libre examen. En effet,

4 Grégoire XVI (1832), «Mirari Vos», Catholicapedia.net. En ligne, http://catholicapedia.net/Documents/Encyclique_Bref_MP/1832-08-15_SS-GREGOIREXVI_Mirari-vos_+_critiques-L-Avenir.pdf, consulté le 21 septembre 2016. 
après deux affaires impliquant universitaires et étudiants ${ }^{5}$, le principe du libre examen est finalement inscrit dans les statuts de l'Université en 1894, institutionnalisant ainsi cette volonté d'autonomie qui a impulsé la création de l'ULB.

Empiriquement, la pilarisation a mené à l'existence de mondes séparés entourant les individus de la naissance à la mort au travers d'institutions spécifiques ${ }^{6}$ :

[Zuilen zijn] min of meer gepolariseerde en met politieke partijen verbonden parallelle netwerken van particuliere organisaties en diensten die elk op grond van een eigen zingevingssysteem werkzaam zijn in meerdere maatschappelijke domeinen en die, in de mate dat de lidmaatschappen samenvallen, kunnen uitgroeien tot aparte collectiviteiten. (Billiet dans Laermans, $1998: 88$ )

En effet, chaque pilier a développé un réseau d'organisations propres : l'enseignement allant de la maternelle au supérieur, des hôpitaux, des mutualités, des assurances, des syndicats et des mouvements de jeunesse. Chaque pilier est également représenté par un parti politique qui l'ancre dans la vie politique en défendant ses intérêts ${ }^{7}$. Le pilier catholique est particulièrement développé, notamment parce que le catholicisme a une place importante dans le pays. Ainsi, au XIX siècle, 99,8 \% des Belges se déclarent catholiques (Dobbelaere, Voyé, 1990). Le pilier forme un milieu de socialisation qui reste persistant jusqu'au début des années 1960 : à la fermeture du Concile Vatican II, on dénombre encore $90 \%$ d'enfants baptisés, $83 \%$ de mariages et $84 \%$ de funérailles célébrés à l'église. De même, jusqu'à cette époque, le clergé et les vocations religieuses ne cessent de croître (Jadoulle, 2003).

Le système de pilarisation est avant tout une configuration de la société civile (Seiler, 1998) qui a une fonction sociale : les piliers cherchent à défendre leur socle confessionnel et les individus qui les composent. Cependant, ce système n'est pas figé et est susceptible d'évoluer.

\section{L'enquête à l'épreuve de la (dé)pilarisation}

La seconde moitié du $\mathrm{XX}^{\mathrm{e}}$ siècle est incontestablement marquée par la « révolution sexuelle»(Simon, 2002 ; Prearo, 2010 ; Giami, Hekma, 2015). Issue des transformations sociétales - démocratisation de l'accès à l'éducation, entrée des femmes sur le marché de l'emploi - cette "révolution » peut se définir selon deux axes. Dans un premier temps, nous entendons les changements survenus du fait de découvertes

\footnotetext{
${ }^{5}$ L'affaire Dwelshauvers en 1890 et l'incident Reclus en 1894 (Daled, 2009).

${ }^{6}$ «Les piliers sont des réseaux parallèles plus ou moins polarisés, liés à des partis politiques et constitués par des organisations et services privés. Leurs activités se situent dans plusieurs domaines sociaux et sont basées sur une philosophie qui leur est propre. Dans la mesure où les affiliations à ces organisations et services convergent, les piliers peuvent se développer jusqu'à devenir des collectivités à part entière. » (Notre traduction)

${ }^{7}$ La question scolaire et la question royale en sont des exemples.
} 
scientifiques ayant influencé les pratiques conjugales et familiales : les contraceptifs (méthode Ogino-Knaus, pilule), les techniques d'avortement, etc. Dans un second temps, la « révolution sexuelle » renvoie à l'individualisation des pratiques sexuelles, c'est-à-dire au « [...] passage d'une sexualité construite par des contrôles et des disciplines externes aux individus à une sexualité reposant sur des disciplines internes " (Simon, 2002: 15). Si les institutions continuent à énoncer des normes en matière sexuelle, ces dernières ne sont plus suivies de manière stricte. Des fidèles utilisaient ainsi la contraception avant la condamnation officielle de l'Église catholique. La parution de l'encyclique Humanae Vitae (1968) confirme cette rupture entre normes et pratiques dans le monde catholique. Ce document papal ${ }^{8}$ déçoit une part des catholiques mais ne remet pas en cause le recours à des pratiques contraceptives (Mulder, 2013). $\mathrm{Au}$ sein de certaines institutions catholiques de protection de la jeunesse, des éducatrices, infirmières ou encore pédagogues ont, par exemple, dans les années 1970 et malgré leurs convictions religieuses revendiquées, participé à la diffusion des moyens de contraception au sein des groupes de vie et rendu plus aisé leur accès.

Avec les changements survenus durant cette période, la fidélité à une institution s'est altérée et c'est la fidélité à soi-même qui commence à être valorisée : «ce qui est en perte de vitesse, c'est l'idée même de loyauté de l'individu vis-à-vis d'une organisation, quelle qu'elle soit» (De Munck, 2009: 52). Les transformations des normes de genre et des pratiques sexuelles survenues lors de la «révolution sexuelle » ont ainsi participé à altérer l'imperméabilité des piliers. En effet, ces transformations les ont transcendés. Si le premier planning familial francophone est laïque (la Famille Heureuse créée en 1962), la planification familiale n’a pas été l'apanage des laïques. Les catholiques ont également répondu à la demande des couples. Représentés par la figure du chanoine Pierre de Locht - symbole d'un catholicisme progressiste -, ils ont su s'intégrer à un réseau d'organisations regroupant des catholiques (le Centre d'éducation à la famille et à l'amour, ainsi que la Fédération belge des centres de consultations conjugales) ${ }^{10}$ et des laïques (la Famille Heureuse) afin de créer la Fédéra-

\footnotetext{
${ }^{8}$ L'encyclique Humanae Vitae est un document papal paru trois ans après la fermeture du Concile Vatican II et qui prend une position officielle sur la régulation des naissances. II rejette toute forme de contraception non naturelle (pilule), alors que de nombreux catholiques espéraient un assouplissement doctrinal faisant suite à l'esprit de modernisation insufflé par le Concile. Voir notamment: Hunt R. E., Hunt J. F., Curran C. E. (dir.) (1969), Dissent in and for the Church: theologians and Humanae Vitae, New York (N.Y.), Sheed and Ward. L'encyclique du pape Paul VI est consultable en ligne sur le site internet du Vatican, http://w2.vatican.va/content/paul-vi/fr/encyclicals/documents/hf_pvi_enc_25071968_humanae-vitae.html, consulté le 15 décembre 2015.

9 Les catholiques ont, de leur côté, ouvert le premier centre de consultations conjugales en 1953, pendant catholique des plannings familiaux. II y a derrière ces créations des enjeux de définition qu'il est nécessaire d'interroger.

${ }^{10}$ Centre d'Animation et de Recherche en Histoire Ouvrière et Populaire (CARHOP), Brainele-Comte, Fonds En Marche, Planning Familial, avortement, contraception (documentation).
} 
tion pluraliste des centres de consultations conjugales ${ }^{11}$. Cette perméabilité aurait contribué à une dépilarisation (tout au moins sur les questions sexuelles), à savoir la reconfiguration des piliers engendrant une restructuration de la société, notamment au travers d'une multiplication des institutions :

Depillarization, in sum, refers to the disarticulation of different spheres of life that were traditionally held together within one pillar, and hence implies a gradual undoing of the segmented organization of the body politic in Belgium. Because the Catholic pillar was historically the most powerful one by far, the process of depillarization has first and foremost amounted to a loss of power by the Catholic Church. (Bracke, Dupont, Paternotte, 2017 : 16)

Partant de l'hypothèse que ces divisions idéologiques n'existaient plus, la question de l'accès aux terrains catholiques ne semblait pas ardue de prime abord. Si la pilarisation a une place dans nos recherches en tant que phénomène historique et politique, d'autant plus que nous réalisons une étude socio-historique, nous avions négligé l'impact actuel de cette structure. Notre expérience de recherche nous a en effet renvoyées à une réalité plus nuancée. Notre position de doctorantes à l'ULB nous a ainsi assignées une identité particulière qu'il convient d'interroger car l'entretien sociologique est une « [...] relation sociale entre deux personnes qui se différencient par leurs caractéristiques sociales, scolaires, sexuelles. C'est un rapport de pouvoir [...]» (Beaud, 1996 : 238). En effet, durant nos entretiens (qu'ils soient informatifs ou qualitatifs), des informateurs du monde catholique nous ont adressé des avertissements bienveillants, tels que "ça aurait été plus facile de faire cette recherche en venant d'une université catholique » ou encore " pourquoi vous vous intéressez aux catholiques ? », qui nous ont rappelées d'où nous venions. Cette assimilation institutionnelle nous renvoyait ainsi aux positions que l'ULB a pu prendre au travers de son histoire sur les questions sexuelles et sur son rapport aux institutions religieuses. L'ULB a ainsi disposé (et dispose toujours) sur son campus, d'un planning familial, ancienne antenne de la Famille Heureuse qui prend son indépendance en 1968. Un premier avortement extrahospitalier y a été pratiqué en 1975, soit 15 ans avant sa dépénalisation en 1990, dans un contexte où l'ULB organisait déjà des colloques dont l'objet était la sexualité libre et responsable. Si Daniel Bizeul se demande, à propos de sa recherche sur le Front National, «[...] ce qui la rend soupçonnable et répréhensible aux yeux d'une partie des lecteurs de sciences sociales [...]» (Bizeul, 2007: 2-3), nous nous demandons ce qui rend nos interlocuteurs suspicieux quant à nos recherches. En effet, ces derniers ne conçoivent pas toujours que, venant de l'ULB, nous puissions nous intéresser au catholicisme sans avoir de préjugés : «il ne faudra pas être dur par principe avec les catholiques », nous dit une informatrice ou encore «les sœurs religieuses ne sont pas

\footnotetext{
${ }^{11}$ Archives générales du royaume (AGR), Bruxelles, Fonds Ligue des Familles, I 553, « 381. Dossier concernant la constitution et le fonctionnement de l'a.s.b.I Centre pluraliste familial. 1969-1976».
} 
des extraterrestres » évoque sur le ton de la plaisanterie une ancienne éducatrice. Dès lors, c'est notre objectivité et nos intentions qui sont remises en cause : un chercheur de l'ULB aurait des a priori sur le monde catholique, et encore davantage quand il le lie à la sexualité. Ceci nous mène souvent à devoir prouver l'absence de jugement dans nos recherches auprès des interlocuteurs catholiques. De fait, cette assignation institutionnelle pose la question de l'accès aux institutions d'obédience catholique.

Toutefois, cette assignation n'est pas unilatérale étant donné que nous participons à la relation d'enquête et que nous attribuons aussi des positions à nos enquêtés et à nos archives:

Avant d'être vécue, la distance sociale est d'abord imaginée et fantasmée ; elle commence à exister avant que le terrain ne commence, au sein d'un contexte social particulier [...]. La distance sociale se construit [...] dans I'anticipation de la méfiance et de la suspicion... (Bonnet, 2008 : 10-11)

Cette projection réciproque nous amène à interroger nos propres prénotions concernant la gestion de la sexualité par le catholicisme. Ne nous identifiant pas au monde catholique, la montée des mouvements conservateurs catholiques ces dernières années a influencé notre perception quant aux données que nous allions recueillir. En effet, l'espace public européen a été la scène d'un processus de politisation des questions de genre et de sexualité sur lesquelles les catholiques - entre autres - se sont mobilisés durant ces dernières années (Paternotte, Piette, van der Dussen, 2016; Bracke, Paternotte, 2016). C'est donc (in)consciemment que nous nous sommes d'abord inscrites dans l'opposition selon laquelle le "progressisme laïque» s'opposerait aux "ténèbres catholiques ». C'est d'ailleurs dans une perspective semblable que s'est construite la figure du chanoine Pierre de Locht en tant qu'acteur progressiste (le chanoine a non seulement défendu la loi dépénalisant l'avortement dès la fin des années 1960 mais aussi l'euthanasie) : il irait à contre-courant de l'Église catholique et serait dès lors une exception confirmant la règle selon laquelle l'Église, à tous les niveaux, est toujours et déjà fermée sur les questions de sexualité. Ce constat est trop simpliste et peut justifier, selon nous, la suspicion des interlocuteurs quant à l'analyse des informations recueillies.

Le fait de parler et de faire parler de sexualité constitue également une question délicate car cela touche à des questions sensibles qui peuvent être jugées tabous et pour lesquelles la prudence semble s'imposer dans les paroles énoncées. De plus, la situation d'enquête elle-même concentre des enjeux de genre (Le Petitcorp, Quintard, 2012) : comment notre identité de femme intervient-elle dans un sujet mobilisant des questions intimes? Faire une enquête sur la sexualité nécessite toujours une méthodologie précise et soignée car « [...] ce qui semble poser problème est la prise de conscience de la violence du dispositif de l'entretien : à savoir susciter - si ce n'est provoquer l'aveu [...] » (Schlagdenhauffen, 2014 : 37). Le retour réflexif sur l'enquête sur la sexualité en France, coordonnée par Nathalie Bajos et Michel Bozon, montre les difficultés de mener des entretiens sur la sexualité. Qu'il s'agisse de thématiques « tabous » (Levinson dans Bajos, Bozon, 2008 : 103), perçues comme « envahissantes par rapport 
à l'intimité de l'enquêté » (Levinson dans Bajos, Bozon, 2008: 103), des thématiques «sensibles » qui dévoilent des informations sur l'intimité des personnes tierces ou encore des thématiques « [...] pour lesquelles certaines réponses seront estimées socialement désirables ou indésirables, notamment dans les domaines où existent des normes claires en matière de comportements ou d'attitudes » (Levinson dans Bajos, Bozon, 2008 : 104). À l'inverse des plannings familiaux où parler de sexualité est une des fonctions de l'institution, les entretiens menés au sein des institutions catholiques de protection de la jeunesse mettent en évidence la difficulté de faire parler le personnel éducatif sur les «dérapages sexuels » entre mineurs ainsi que sur la question de la pédophilie. Cette dernière particulièrement controversée suite aux différentes affaires auxquelles l'Église a été confrontée, s’inscrit en effet dans des représentations collectives locales amalgamant catholiques et pédophilie. Ces thématiques sont souvent occultées et délicates à aborder en situation d'entretien. Du côté de l'enquêteur, des stratégies de contournement peuvent être adoptées. Il est possible d'entamer l'entretien sur une thématique n'abordant pas frontalement la question de la sexualité. C'est par exemple le cas du thème de la mixité dans les institutions de protection de la jeunesse, qui permet une entrée sur les questions sexuelles : les enquêtés abordent d'eux-mêmes les questions sexuelles, thématique découlant logiquement pour eux de la mise en présence des deux sexes. Les enquêtés peuvent quant à eux ressentir de la gêne dans le dévoilement et la confidence d'informations sensibles qui les concernent ou qui impliquent d'autres individus. C'est le cas des questions de pédophilie et de «dérapages sexuels » : si les interlocuteurs reconnaissent l'existence de ce phénomène, ils le renvoient dans l'altérité : «oui, cela existe, mais pas partout » dit un éducateur - et pas ici, sous-entendent-ils avant de changer de sujet. Malgré ces difficultés, il est possible et nécessaire d'accéder à un terrain qui semble $a$ priori fermer ses portes à notre approche.

\section{Dépasser la logique des piliers}

La distance qui se crée entre enquêteurs et enquêtés, si elle semble indépassable et source d'angoisse pour le chercheur, peut également être un matériau d'enquête en soi. François Bonnet, citant Sudhir Venkatesh, y voit ainsi une partie même du terrain sociologique :

L'interaction entre l'ethnographe et ses informateurs est elle-même potentiellement révélatrice des propriétés locales de la structure sociale et peut être aussi creusée pour éclairer les questions de recherche. Le questionnement méthodologique ne doit pas se résumer à décrire à quel point on a réussi à « accéder » au terrain. (Bonnet, $2008: 4-5$ )

Ainsi, l'altérité décrite entre les catholiques et les laïques, sur un terrain qui s'intéresse à la réduction de cette différence, peut nous aider à formuler des hypothèses sur notre objet de recherche : si la logique de dépilarisation a déjà été mise en 
avant, cette assimilation institutionnelle révèle-t-elle des résidus de pilarisation? Aussi, au-delà de la persistance des piliers, la pilarisation ne peut-elle pas être envisagée comme un outil permettant une utilisation opportuniste ou instrumentale du concept : une catégorie à laquelle il est aisé de faire appel pour expliquer des dissensions.

Si les obstacles empiriques nous renseignent sur la persistance des piliers et nous incitent à continuer dans la voie de la socio-histoire afin de comprendre nos difficultés, il est néanmoins nécessaire de dépasser cette assimilation institutionnelle afin de rendre compte des complexités individuelles. Si nous devons, en tant que chercheuses, réussir à donner une image plus détachée de celle qu'induit notre étiquetage institutionnel dans un contexte de pilarisation, il en va de même pour les personnes que nous rencontrons sur notre terrain. Ainsi, le récit de Gisèle Bastin ${ }^{12}$, formatrice au Centre National de Pastorale Familiale dans les années 1960 et se revendiquant catholique, rend compte de cette dichotomie entre institutions et individus. Devenue conseillère conjugale à la FBCCC (Fédération belge des centres de consultations conjugales) d'obédience catholique dès sa création à la fin des années 1960, elle fait personnellement le trajet jusqu'aux Pays-Bas afin d'accompagner des femmes pour qui l'avortement était une nécessité, contre l'avis officiel de son institution d'appartenance. Cela met également en lumière l'importance des sources orales et des récits de vie: les données institutionnelles ne rendent pas compte de la complexité d'un problème politique et social. Il est nécessaire de s'intéresser aux différents niveaux sociologiques qui le composent. Dès lors, la question est de savoir comment accéder à un terrain qui nous résiste du fait de la structure sociale dans laquelle ce terrain est réalisé ?

Pour éviter de s'entendre dire que nous ne connaissons rien à la religion catholique la présentation de soi et la réflexivité sont les atouts des chercheurs :

[...] «se fondre dans le terrain » (réduire la distance) n'est de toutes façons ni «possible» ni «nécessaire ». En s'appuyant sur une conception de la culture comme «boite à outil » et répertoire pour l'action (Swidler 1986), on peut comprendre qu'il est tout à fait à la portée du sociologue déterminé à faire son terrain d'acquérir petit à petit des «compétences culturelles» qui lui permettent d'interagir de façon fluide avec ses interlocuteurs. (Bonnet, 2008 : 13-14)

Dans la situation d'entretien, le renvoi à notre identité de chercheuses de l'ULB est le plus courant. Que l'entretien soit exploratoire ou qualitatif, une certaine retenue s'impose à nous à son début, afin d'installer un climat de confiance. Laisser s'exprimer librement l'interlocuteur, éviter dans un premier temps de mettre en avant son propre positionnement, « laisser en suspens des valeurs jugées essentielles »

${ }^{12}$ Archives et Musées de la Littérature, Bruxelles, Fonds Gisèle Bastin, MLPA 00256, «Le centre pluraliste et les plannings familiaux ». 
(Bizeul, 2007 : 3), adopter une attitude dépourvue de jugement de valeur, reconnaître ce qui différencie l'autre de soi, être empathique et à l'écoute et réagir aux propos énoncés en allant d'abord dans leur sens, constituent des stratégies qui, durant l'entretien, peuvent participer à rendre moins importante, voire faire disparaitre l'identité potentiellement "problématique» à laquelle nous sommes généralement renvoyées. En effet, « les assignations de place ne jouent pas nécessairement au long cours, elles agissent de manière fluctuante» (Monjaret, Pugeault, 2014: 83). Ceci n'empêche pas, une fois la relation de confiance plus ou moins établie avec les interlocuteurs, de revenir sur certains éléments évoqués lors des entretiens et progressivement prendre parti et défendre ses valeurs. Il s'agit davantage de jouer sur notre propre identité et sur la distance sociale qui peut nous séparer ou nous rapprocher de l'enquêté : le fait d'être étrangère, par exemple, permet de sortir de la logique de pilarisation et de prendre des distances à son égard, comme l'explique Pierre-Noël Denieuil (2014) : « c'est à ce titre que ma distance comme étranger renforce ma proximité et l'empathie avec mes interlocuteurs, car je suis "autre” »(p.121). Des intérêts communs peuvent également permettre d'accéder à des données : dépasser l'assignation est possible en créant des «terrains d'entente qui rendent possibles les questions des un(e)s et les réponses des autres » (Mallon, 2014 : 92). En effet, l'importance du travail de mémoire est ainsi un argument utile afin d'obtenir des archives auxquelles il est difficile d'avoir accès. Les institutions deviennent dès lors complices du travail réalisé, et la recherche se développe sur une base d'échange plutôt que sur une rivalité. Cela participe également à la création d'un réseau de recherche qui permet de sortir de son pilier d'origine. Faire une socio-histoire d'institutions catholiques dans une logique « transpilier » est donc souhaitable et possible, à condition de faire le travail réflexif sur la posture à adopter.

\section{Conclusion}

La religion et la sexualité sont tous deux des sujets jugés « tabous »; lier les deux peut donc créer un objet de recherche difficile à appréhender. La situation d'enquête crée une suspicion engendrée par l'articulation de ces deux thématiques et nous met parfois face à la nécessité de justifier une telle recherche : pourquoi travailler sur le rapport à la sexualité des catholiques ? Est-ce une remise en cause des compétences de gestion des questions sexuelles des institutions catholiques ? Est-ce un moyen de démontrer la distance qui peut les séparer de «nous » ? Il s'agit au contraire de combler un manque: les institutions catholiques n'ont pas toujours fait l'objet de recherches systématiques. Ainsi, les plannings familiaux laïques ont attiré l'attention des chercheurs, laissant de côté la prise en charge des couples par des organisations catholiques. La gestion de la sexualité au sein d'institutions catholiques de protection de la jeunesse reste également à explorer. Cette remise en cause de la légitimité d'enquêter sur la gestion de la sexualité par des catholiques doit donc être interrogée et débou- 
cher sur l'analyse de l'accès au terrain (qu'il soit constitué d'archives ou d'entretiens). En effet, la situation d'entretien nous apporte déjà des éléments sur la (dé)pilarisation, concept et processus que nous voulons par ailleurs interroger lors de nos thèses. Elle nous apprend que si les écrits des chercheurs décrivent un processus de dépilarisation entamé dès les années 1960, la Belgique ne peut empiriquement pas s'étudier sans prendre en compte les résidus de pilarisation qui la composent.

Avant d'arriver sur le terrain, nous pensions en effet que l'appartenance à un pilier ne déterminerait pas notre accès au terrain. Cependant, l'appartenance institutionnelle exerce bel et bien un impact sur la réalisation de notre étude en ce que nos interlocuteurs nous assignent à une position avec laquelle il faut pouvoir jouer. La suspicion dont nous sommes témoins et parties nous montre que notre hypothèse de départ, à savoir que l'individualisation de la sexualité et plus largement la «révolution sexuelle » avaient annihilé les effets de la pilarisation sur de telles questions, est donc à considérer avec circonspection. De même, l'idée que la gestion de la sexualité était différente entre catholiques et laïques doit être précisée : les catholiques ne sont pas ignorants en matière de prise en charge des questions sexuelles. Cette réflexion méthodologique et analytique de notre présence sur le terrain permet aussi d'imaginer une analyse qui irait au-delà des piliers et qui rendrait compte de la complexité des positions de l'Église face aux normes sexuelles.

\section{Bibliographie}

Bajos N., Bozon M. (dir.) (2008), Enquête sur la sexualité en France. Pratiques, genre et santé, Paris, La Découverte.

BEAUD S. (1996), « L'usage de l'entretien en sciences sociales. Plaidoyer pour l'“entretien ethnographique” », Politix, vol. 9, n³ 35, p. 226-257.

BizeUL D. (2007), «Des loyautés incompatibles. Aspects moraux d'une immersion au Front National », SociologieS.

BONNET F. (2008), "La distance sociale dans le travail de terrain: compétence stratégique et compétence culturelle dans l'interaction d'enquête », Genèses, $n^{\circ} 73$, p. 57-74.

Bracke S., Dupont W., Paternotte D. (2017), «"No prophet is accepted in his own country": Catholic anti-gender activism in Belgium », in R. KuHAR, D. PATERNotTE (dir.), Anti-Gender Campaigns in Europe: Mobilizing Against Equality, Londres, Rowman \& Littlefield.

Bracke S., Paternotte D. (2016), « Habemus Gender! The Catholic Church and "Gender Ideology" », Religion erGender, vol. 6, n² 2. 
CRISP (1974), « L'évolution du “monde catholique” en Belgique depuis 1968 : le devenir de la pratique religieuse ", Courrier hebdomadaire du CRISP, vol. 38, n 664, p. 1-28.

DAled P. (2009), « Le libre examen : la vie d'un principe » in D. JONCKHEERE, J. LEMAIRE (dir.), 1834. L’ULB fête ses 175 ans, Bruxelles, Éditions Espace de Libertés (« La pensée et les hommes»).

De Ganck J., D’Hooghe V. (dir.) (2013), Regards sur le sexe, Bruxelles, Éditions de l’Université de Bruxelles («Sextant»).

De Maeyer J., Put E., Roegiers J., Tihon A., Vanden Bosch G. (2009), L'archidiocèse de Malines-Bruxelles. 450 ans d'histoire. Volume 2. L'Église populaire dans l'archidiocèse. Une Église "libre» dans une société moderne 1802-2009, Louvain, Uitgeverij Halewijn.

DE MuncK J. (2009), «Que reste-t-il des clivages en Belgique ?», La revue nouvelle, $\mathrm{n}^{\circ} 10$, p. 31-65.

Defosse P. (dir.) (2005), Dictionnaire historique de la laïcité en Belgique, Bruxelles, Fondation rationaliste/Éditions Luc Pire («Voix de l'Histoire »).

Deloye Y. (2007), Sociologie historique du politique, Paris, La Découverte.

DeNiEuil P. (2014), "Rencontre d'un chercheur étranger et d'entrepreneures tunisiennes», in A. Monjaret, C. Pugeault (dir.), Le sexe de l'enquête. Approches sociologiques et anthropologiques, Lyon, ENS Éditions, p. 113-126.

DesCAMPS F. (2005), L'historien, l'archiviste et le magnétophone. De la constitution de la source orale à son exploitation, Paris, CHEEF.

Dobbelaere K., Voye L. (1990), « From Pillar to Postmodernity: The Changing Situation of Religion in Belgium », Sociological Analysis, vol. 51, p. 1-13.

Dobbelaere K., Voye L. (2012), «Portrait du catholicisme en Belgique », in A. PereZAgote (dir.), Portraits du catholicisme. Une comparaison européenne, Rennes, Presses universitaires de Rennes, p. 11-61.

FANIEL J., VANDAELE K. (2011), « Histoire de la Centrale générale des syndicats libéraux de Belgique (CGSLB) », Courrier hebdomadaire du CRISP, n² 2123-2124, p. 1-53.

FLANDRIN J.-L. (2006), L'église et la contraception, Paris, Imago.

Giami A., Hekma G. (dir.) (2015), Révolutions sexuelles, Paris, La Musardine.

Houdailles J. (1980), « La baisse de la fécondité en Belgique au XIX ${ }^{\mathrm{e}}$ siècle », Population, vol. $35, \mathrm{n}^{\circ} 2, \mathrm{p} .450-456$.

HunT R. E., HunT J.-F., CuRRAN C. E. (dir.) (1969), Dissent in and for the Church: theologians and Humanae Vitae, New York, Sheed and Ward. 
JADOUlle J.-L. (2003), Chrétiens modernes? L'engagement des intellectuels catholiques " progressistes » belges de 1945 à 1958 à travers "La Revue Nouvelle », "La Relève » et l'édition belge de "Témoignage chrétien », Louvain-la-Neuve, Éditions AcademiaBruylant.

LAERMANS R. (1998), Godsdienst en Kerk in een geseculariseerde samenleving. Een keuze uit het werk van Karel Dobbelaere, Louvain, Universitaire Pers Leuven.

LANGLoIS C. (2010), «Sexe, modernité et catholicisme. Les origines oubliées », Esprit, $\mathrm{n}^{\circ} 2$, p. 110-121.

Le Petitcorp C., Quintard M. (2012), «Jeux et enjeux dans les relations d'enquête: expériences de terrain et de dominations croisées », e-Migrinter, vol. 9, p. 91-106.

Levinson S. (2008), "La place et l'expérience des enquêteurs dans une enquête sensible », in N. Bajos, M. Bozon (dir.), Enquête sur la sexualité en France. Pratiques, genre et santé, Paris, La Découverte, p. 97-113.

LIJPharT A. (1977), Democracies in Plural Societies: A comparative Exploration, New Haven, Yale University Press.

Mallon I. (2014), "Une sociologue et des résident(e)s en maison de retraite. Des relations d'intimité familiale et de séduction courtoise», in A. MONJARET, C. Pugeault (dir.), Le sexe de l'enquête. Approches sociologiques et anthropologiques, Lyon, ENS Éditions, p. 85-97.

Martiniello M., ReA A. (2004), « Piliers, minorités ethnique et affirmative action en Belgique. Les minorités ethniques sont-elles solubles dans le pluralisme belge ?», in M. MARTINiello, A. ReA (dir.), Affirmative action. Des discours, des politiques et des pratiques en débat, Bruxelles, Éditions Academia-Bruylant.

MCCLORY R. (1998), Rome et la contraception: histoire secrète de l'encyclique Humanae Vitae, Paris, Éditions de l'atelier.

Monjaret A., Pugeault C. (2014), « Introduction. En quête de genre » in A. Monjaret, C. PugEault (dir.), Le sexe de l'enquête. Approches sociologiques et anthropologiques, Lyon, ENS Éditions, p. 5-17.

Monjaret A., Pugeault C. (2014), «Le travail du genre sur le terrain. Retours d'expériences dans la littérature méthodologique en anthropologie et en sociologie», in A. Monjaret, C. Pugeault (dir.), Le sexe de l'enquête. Approches sociologiques et anthropologiques, Lyon, ENS Éditions, p. 18-45.

Mulder R. (2013), Seks en de kerk. Hoe Nederland zijn kuisheid verloor, Amsterdam, Atlas contact.

NoIRIEL G. (2008), Introduction à la socio-histoire, Paris, La Découverte. 
NoOnan J. (1965), Contraception: a history of its treatment by the Catholic theologians and canonists, Harvard, Press of Harvard University.

Paternotte D., PietTe V., VAn DeR Dussen S. (dir.) (2016), Habemus gender! Déconstruction d'une riposte religieuse, Bruxelles, Éditions de l'Université de Bruxelles (« Sextant»).

Prearo M. (dir.) (2010), « Politiques de la libération sexuelle», Genre, sexualité er société, $\mathrm{n}^{\circ}$ 3, p. 1-5.

ROCHEFORT F., SANNA M.-E. (2013), Normes religieuses et genre: mutations, résistances et reconfiguration, $\mathrm{XIX}^{e}-\mathrm{XXI}{ }^{e}$ siècle, Paris, Armand Colin.

SChlagdenhauffen R. (2014), "Parler de sexualité en entretien. Comment rendre publics des propos privés », Hermès. La Revue, n 69, p. 34-38.

SEILER D.-L. (1998), La vie politique des Européens: introduction aux pratiques démocratiques dans les pays de l'Union européenne, Paris, Economica.

SEVEGRAND M.. (1995), Les enfants du bon Dieu. Les catholiques français et la procréation au $\mathrm{XX} \mathrm{X}^{e}$ siècle, Paris, Albin Michel.

Simon P. (2002), « Révolution sexuelle ou individualisation de la sexualité ? Entretien avec Michel Bozon », Mouvements, $\mathrm{n}^{\circ}$ 20, p. 15-22. 